\title{
Jump Ship, Shift Gears, or Just Keep on Chugging: Assessing the Responses to Tensions between Theory and Evidence in Contemporary Cosmology
}

\author{
Siska De Baerdemaeker \& Nora Mills Boyd
}

\begin{abstract}
When is it reasonable to abandon a scientific research program? When would it be premature? We take up these questions in the context of a contemporary debate at the border between astrophysics and cosmology, the so-called "small-scale challenges" to the concordance model of cosmology $(\Lambda \mathrm{CDM})$ and its cold dark matter paradigm. These challenges consist in discrepancies between the outputs of leading cosmological simulations and observational surveys, and have garnered titles such as the Missing Satellites, Too Big To Fail, and Cusp/Core problems. We argue that these challenges do not currently support a wholesale abandonment or even modification of cold dark matter. Indeed, the nature of the challenges suggests prioritizing the incorporation of known physics into cosmological simulations.
\end{abstract}

Keywords: theory change; anomalies; dark matter; small-scale challenges; simulation; cosmology

\section{Highlights:}

\section{- Small-scale challenges}

- Is a revolution nigh?

- Patience, stay the course

\section{Introduction}

For many decades, philosophers of science have grappled with the methodology of theory change (e.g. Kuhn 1962, Toulmin 1972, Laudan 1977, Hacking 1981, Darden 1991, and Thagard 1993). When should theoretical changes accommodate empirical anomalies? When would that be 
unhelpfully ad hoc? When is it reasonable to abandon a scientific research program? When is it unreasonable not to abandon one?

Imre Lakatos (1978) formulated one of the subtler of the well-known philosophical approaches to these questions. Lakatos' view on the methodology of scientific research has the benefit of emphasizing the evolution of scientific theories. For Lakatos, a research programme consists in a "hard core", which is for all intents and purposes immune to falsification in virtue of the negative heuristic of the programme, and a "protective belt" of auxiliary hypotheses that is open to revisions in response to the pressures of empirical tests (the positive heuristic). "Progressive" (i.e. successful) research programmes are ones that continue to produce novel predictions after revisions, at least some of which are borne out by new evidence. In contrast, degenerating research programmes fail to be progressive in this way.

Lakatos' approach is more lenient than would be a hard-nosed Popperian falsificationism (that Popper himself did not endorse) since Lakatos allows that the progress of a successful programme may be intermittent—not every novel prediction must be immediately corroborated. Lakatos allows that it may be rational to place prima facie refutations of a research programme "into some temporary, ad hoc quarantine" and get back to work $(1978,58)$.

The suggested permissibility of quarantining prima facie refutations raises several critical questions. Under what conditions is it acceptable to retain allegiance to a programme facing prima facie refutations? How long can such "temporary" quarantine be rationally maintained? How many difficulties can be quarantined before it must be admitted that there is a serious problem with the research programme? Without answers to such questions, scientists can glean little specific practical guidance from the Lakatosian framework in the circumstances where such guidance would be most 
useful-in making decisions about where to focus research efforts and how to prioritize them in the face of accumulating anomalies. Indeed the Lakatosian approach has been criticized on the grounds that the progressive/degenerative status of a research programme can only be judged in retrospect (Chall 2020, 18). This has led Chall (2020) for example, to combine a version of Lakatos' approach (modified to include models) with a Laudan-inspired appraisal of problem-solving successes and capacities. ${ }^{1}$ We share Chall's criticism of the Lakatosian approach-we would like a contemporaneous way to appraise research programmes. ${ }^{2}$

We take it as a premise that practically useful in situ guidance for appraising research programmes in light of accumulating anomalies should avoid sliding into the Kuhnian picture of theory change on the model of religious conversion. One wants to retain, to the extent possible, useful heuristics for reasoning through theory change. By this we do not mean to endorse the much-discussed value-free ideal in science (see e.g. Rudner 1953, Jeffrey 1956, Longino 1990, and Douglas 2009). ${ }^{3}$ We believe that the co-existence of values and reason is possible, indeed necessary in science, and do not deny that values can play a role in reasoning across theory change. ${ }^{4}$ That said, our argument does rest on the following two assumptions: 1) a contemporaneous method for rationally prioritizing research strategies in the face of apparent anomalies would be valuable, and 2)

\footnotetext{
${ }^{1}$ We are grateful to an anonymous referee for pointing us to this reference.

${ }^{2}$ We part ways with Chall's move to a hybrid Lakatos-Laudan view. Laudan's approach appraises theories not merely with respect to their successes in solving empirical problems, but also in solving theoretical/conceptual problems. Chall applies this hybrid framework for instance, to work in appraising the status of Supersymmetry, appealing the problem of "naturalness" (21). We believe that such an approach strays too far from the empirical, and thus are concerned in the present paper with supplementing a roughly Lakatosian approach with further heuristics that allow for contemporaneous appraisal of research programs, while retaining the focus of theory-appraisal on empirical adequacy.

${ }^{3}$ We thank an anonymous referee for pressing us to clarify this point.

${ }^{4}$ We thank an anonymous referee for raising the concern that our argument ignores the inherently paradigm-internal nature of values. We do not deny that values invoked in scientific reasoning have evolved over the history of science (see for example Daston \& Galison 2007, and Franklin 2013). However, we also believe that this fact alone does not negate the possibility of reasoning across theory change. In case of the small-scale challenges, for example, we believe that the Kuhnian picture of incommensurability is too broad to capture the content of the debate. We take the debate about the small-scale challenges to be much more local, as will become clear below.
} 
articulating such a method is not in principle impossible. This first assumption is just the criticism of Lakatos that we already cited from Chall above, and we suspect is widely held. We hope that our argument below is an existence proof in support of the second.

In the present work, we take up these questions about the methodology of theory change in the context of a contemporary debate at the border between astrophysics and cosmology, the so-called "small-scale challenges" to the cold dark matter paradigm. We take an opposite approach to that of Popper, Lakatos or Kuhn: rather than focusing on the general question of theory change ("how should a research programme respond to anomalies?"), we will focus on a specific debate ("how should the current concordance model of cosmology be modified, if at all, in response to the small-scale challenges?") and approach the general question from the ground up. We focus upon the small-scale challenges debate in particular as a methodological test case because this debate is currently live and ongoing. Intervention by philosophers of science thus may be useful in active decision-making in this field of research. Moreover, such intervention could add to the budding philosophical literature on computer simulations in cosmology and astrophysics (see also Gueguen Forthcoming; Smeenk \& Gallagher Forthcoming).

We argue that in this context, specific heuristics recommend a compelling hierarchy of priorities to follow. In particular, the small-scale challenges do not currently support a wholesale abandonment of cold dark matter or even a modification of the cold dark matter hypothesis (see Sections 3.1 and 3.2 below). Indeed, the nature of the challenges suggests prioritizing the incorporation of known physics into cosmological simulations. Although our main focus will be on the small-scale challenges debate, we believe that some of our arguments can have a more 
widespread application in scientific practice, without pretending to be a general account of theory change (see Sections 6 and 7).

Section 2 briefly introduces the small-scale challenges. In Section 3 we classify the different possible solutions to these challenges into three categories, and argue for our preferred approach in Section 4. Section 5 considers different possible futures for astrophysics and cosmology with respect to the challenges, and discusses recommendations for each of these scenarios according to the position we defend. In Section 6 we respond to potential objections to our argument motivated by methodological pluralism and the recent work on the epistemic value of risky research.

\section{Small-scale challenges in cosmology}

The small-scale challenges in contemporary cosmology consist in a handful of tensions between theoretical predictions and empirical evidence. Theoretical predictions are based on the current concordance model of cosmology, the "Lambda Cold Dark Matter" model ( $\Lambda \mathrm{CDM}) . \Lambda \mathrm{CDM}$ models our universe using the resources of general relativity in concert with other physical theories that inform our understanding of the composition and evolution of the universe. According to $\Lambda \mathrm{CDM}$, the universe is best described by an FLRW-metric (i.e. is homogeneous and isotropic) on large scales. Its energy density consists of about 5\% baryonic matter, $26 \%$ dark matter-CDM—and $69 \%$ dark energy- $\Lambda$ (for a theoretical introduction see Peacock 2015, for recent empirical results see Planck Collaboration 2018). To model structure formation, $\Lambda$ CDM helps itself to purely adiabatic scalar primordial perturbations with a power-law spectrum, that eventually seed large-scale structure-the "cosmic web" of galaxy clusters. On large scales, this model accords well with empirical evidence, and it furnishes a satisfactory narrative of cosmic evolution, stretching from the formation of the lightest elements, through the decoupling of the Cosmic Microwave Background 
(CMB) radiation, to the formation of dense filaments of matter housing clusters of galaxies capable of star formation.

The present paper will not discuss the, admittedly philosophically fascinating, collection of issues surrounding dark energy. Instead, our focus will be on the dark matter component. By now there are many and varied sources of empirical evidence consistent with dark matter. On astrophysical scales (galaxies and clusters of galaxies), crucial observations supporting a dark matter component include Zwicky's observations of velocity dispersions in the Coma cluster (Zwicky 1933/2009), Rubin's galaxy rotation curves (Rubin \& Ford 1970), and the more recent observation of the Bullet Cluster, a merging event between two galaxies where the gravitational and the baryonic mass are separated from one another and have been imaged separately using weak gravitational lensing and X-ray observations, respectively (Clowe et al. 2006). On cosmological scales, theories of structure formation require an additional source of mass in order to provide sufficient gravitational collapse to reproduce the sort of cosmic structure mapped by large-scale observational surveys of galaxy clusters and of the cosmic microwave background radiation (White \& Rees 1978). Reproducing the observed CMB power spectra also requires including a dark matter component in the cosmological model (Planck Collaboration 2018). This (non-exhaustive) collection of empirical results has been used to place constraints on the nature of dark matter. So far, to be consistent with the evidence, dark matter cannot be baryonic, it cannot interact electromagnetically, there are strict limits on its coupling through the strong interaction, and its self-interaction cross-section must be limited. Yet many further questions remain about the specific nature of this mysterious ingredient that evidently makes up about a quarter of the entire energy density of the universe today, and which 
played a major (if not entirely defining) role in the formation and evolution of structure in our cosmos.

In an attempt to learn more about dark matter, astrophysicists and cosmologists are currently interested in comparing predictions from $\Lambda \mathrm{CDM}$ and observations at high resolution-i.e. relatively small spatial scales, cosmologically-speaking. Predictions derived from $\Lambda \mathrm{CDM}$ often come in the form of computer simulation outputs when solving the relevant equations analytically is impossible. Cosmic structure formation is simulated and then the results of those simulations are compared with the results of large-scale observational surveys of galaxies, clusters, and superclusters. In the early days, the physics that cosmological simulations modeled was relatively austere; they modeled either massive particles or fluid volumes, and the gravitational interactions between them - see for example the Millennium Run (Springel et al. 2005). The reasoning behind this approach was that, on the largest scales, the strength of the gravitational interaction dominates over all others, and it was thought that other physical processes could be neglected. Neglecting other physical processes was also crucial to keep the computational costs of the early large-scale cosmological simulations under control.

On smaller scales, it turns out that there are a handful of substantive points of disagreement between the matter and gravitation-only simulations and the results of empirical surveys (Bullock \& Boylan-Kolchin 2017). Some of these discrepancies have become so widely accepted that they have garnered special names. Here we mention three: Missing Satellites, Cusp/Core, and Too Big to Fail. The Missing Satellites problem consists in the fact that simulations of the formation of Milky-Way sized galaxies predict that they should be surrounded by a host of satellite dwarf galaxies, yet observational evidence shows fewer such galaxies by an order of magnitude. The Too Big To Fail 
Problem is linked to the Missing Satellites problem. One could argue that the reason why surveys have not found the remaining satellites of the Milky Way is that those satellites do not contain any baryonic matter, i.e. are just dark matter halos. However, it turns out that the predicted satellite galaxies would be too big to not contain baryonic matter. They are, in other words, too big to fail at having star formation. Finally, the Cusp/Core problem consists in the fact that cosmological structure formation simulations predict a particular density profile for dark matter halos. Specifically, halos are expected to have a 'cusped' density profile, where the density increases rapidly at decreasing radii. Observations of galaxy rotation curves, however, lead to the conclusion that the halo density profiles are 'cored', i.e. that the density profiles do not rapidly increase at the center of galaxies.

We focus on these three tensions because we take them to be the three that are widely considered as genuine 'challenges', where theoretical prediction and empirical results disagree. We recognize, however, that some researchers consider other sets of observations as similarly challenging to $\Lambda \mathrm{CDM}$ : a set of regularities collectively called 'MOND phenomenology'. ${ }^{5} \mathrm{MOND}$ originally arose as a rival explanation (to dark matter) of the galaxy rotation curves observed by Rubin and collaborators in the 1970s and 1980s. Following a proposal by Milgrom (1983), MOND claims that the rotation curves can be explained by modifications to the laws of Newtonian dynamics. This was considered preferable by some to introducing a new "hidden", non-baryonic type of matter that supposedly resided at the outskirts of galaxies. In addition to the galaxy rotation curves, there are other regularities that are "generally expected in MOND" (Bullock \& Boylan-Kolchin 2017, 368). The Baryonic Tully-Fisher Relation, a correlation between the baryonic

\footnotetext{
${ }^{5}$ We would like to thank an anonymous referee for pressing us on this point.
} 
mass and the asymptotic rotational velocity of a disk galaxy, is probably the most well-known example. Given the context of this special issue, we briefly summarize our position on MOND phenomenology.

We do not consider it a problem for $\Lambda \mathrm{CDM}$ if $\Lambda \mathrm{CDM}$ fails to 'explain' these correlations in the way that the MOND formalism claims to. Rather, we agree with Massimi $(2018,34)$ that if simulations retrieve the astrophysical data underpinning these correlations, that is "success enough and must count as success enough for $\Lambda \mathrm{CDM}$ ". We would, however, consider the elements of MOND phenomenology to constitute genuine challenges for $\Lambda \mathrm{CDM}$ if the predictions derived from the concordance model turned out to be inconsistent with the various correlations that are derivable from astrophysical observations. In that case, and only in that case, would we consider MOND phenomenology to be challenges on par with the aforementioned small-scale challenges. Following Bullock \& Boylan-Kolchin (2017), we do not yet take this to be the case.

The small-scale challenges present an interesting case for philosophy of science. We have here prima facie disagreement between predictions made using an otherwise successful model and certain empirical results. These challenges are widely considered issues that need to be addressed. Are there reasonable methodological prescriptions that could guide researchers in choosing how to proceed in the face of these challenges? One might wonder whether these small-scale challenges should be put in a Lakatosian "quarantine" or if they are well-established and enduring enough to spell serious trouble for the cold dark matter research programme, and what features of the scenario would motivate a choice one way or the other. If one's primary aim is descriptive fidelity to scientific practice, one might just want to wait around to see whether branches of cosmological research that quarantine the challenges or those that make theoretical modifications in response to the challenges 
end up being degenerating in the Lakatosian sense. However, thinking prescriptively, one could also be curious if there are good arguments now, in the moment of decision, for pursuing a particular approach rather than alternatives. In the next section, we lay out the available choices by describing research that has developed in response to the small-scale challenges.

\section{Solving the small-scale challenges}

Three types of approaches can be taken to resolving the small-scale challenges. We call these 1) jump ship, 2) switch gears, and 3) keep on chugging. To jump ship is to abandon one's research programme. To switch gears is to shift focus to alternatives within one's research programme previously considered to be less promising. To keep on chugging is to stay the courseto try to resolve existing challenges using the current approach.

\subsection{Jump ship: reject dark matter entirely}

One minority view argues that dark matter should be rejected entirely in light of the small-scale challenges. This is a radical approach; it would constitute a complete overhaul of $\Lambda$ CDM. Scientists do not usually jump ship unless there is a new, promising, "ship" to jump into. Some proponents of modified Newtonian dynamics (MOND) have taken this possibility seriously-arguing that the challenges to $\Lambda \mathrm{CDM}$ are dire enough to support serious consideration of competing theoretical (MONDian) approaches. McGaugh (2014) for instance, has described the difference between MOND and $\Lambda \mathrm{CDM}$ as "a tale of two paradigms", which could be interpreted as implying that MOND should be viewed as a genuine competitor to $\Lambda \mathrm{CDM}$, worthy of the attention of the physics community.

Today, it is safe to say that MOND has not been viewed favorably by most of the cosmology community. First, as Massimi (2018) explains, MOND currently fails to accurately account for 
cluster-phenomenology_-specifically the presence of collisionless missing mass in the Bullet Cluster. The addition of dark matter to the MOND paradigm is one of several proposed solutions. This is illustrative of a more general attitude adopted by even some of those most devoted to MOND: they do not advocate abandoning $\Lambda \mathrm{CDM}$ outright, even in light of the small-scale challenges. Instead of calling for a rejection of $\Lambda \mathrm{CDM}$, for instance, Famaey \& McGaugh (2012, section 10) suggest that “it would probably be a mistake to persistently ignore the fine-tuning problems for dark matter and the related uncanny successes of the MOND paradigm on galaxy scales, as they could very plausibly point at a hypothetical better new theory". They leave open the possibility that problems that $\Lambda \mathrm{CDM}$ faces might be best solved by a combination of dark matter (in the form of compact non-luminous baryonic matter, hot dark matter like neutrinos, or exotic dark fields) and MOND.

Second, Massimi (2018) points out that MOND currently fails to account for large-scale structure formation and CMB phenomenology. This is because MOND is currently not unified with general relativity. Until recently, Bekenstein's TeVeS-approach was the most likely candidate to achieve the MOND-GR unification, but LIGO's neutron star merger data yielded conclusive evidence excluding TeVeS (Boran et al. 2018). Other proposed extensions of MOND have received substantive criticism from other MONDians (Massimi 2018, 36), revealing a lack of theoretical unity.

While abandoning $\Lambda \mathrm{CDM}$ on the basis of the small-scale challenges (for instance, in favor of MOND) is a logical possibility, it has neither the substantive support of proponents in cosmology, nor independent reasons to recommend it. Jumping ship in this situation would be premature: MOND does not constitute a working alternative, especially without an explicit unification with GR and a recovery of large-scale cosmological phenomenology. Furthermore, as we will argue below, it is not actually clear that the small-scale challenges present insurmountable anomalies to $\Lambda$ CDM. In 
general we suspect that the following is a reasonable strategy: do not jump ship unless 1) your current ship is malfunctioning, and 2) the alternative ship to which you intend to jump is at least as seaworthy as your current one. For the remainder of this paper, we will therefore consider more moderate, and we think more realistic, responses to the small-scale challenges.

\subsection{Switch gears: modify the dark matter bypothesis}

Alternatively, one might maintain the central tenet of the dark matter hypothesis: that the observations described in Section 1 provide evidence compatible with the existence of a non-baryonic matter contribution to the energy density of the universe. Within the constraints derived from these observations, such more cautious revisionary approaches rethink the properties of dark matter, usually by postulating more interactions for dark matter than the cold dark matter hypothesis does.

One example of such a cautionary modification is self-interacting dark matter (SIDM). SIDM was proposed by Spergel and Steinhardt (2000) specifically in response to the Cusp/Core problem. As the name suggests, it proposes a non-negligible self-interaction between dark matter particles. Astrophysical and cosmological considerations put quite stringent constraints on the possible size of the interaction cross-section (see Tulin \& Yu 2017 for a review). For example, the mean free path of the dark matter particles needs to be large enough so that SIDM retains the success of CDM on larger scales, but small enough so that the particles do not move through a halo without interacting. The dark matter particles' self-interaction must change halo evolution compared to CDM so that there is any hope of empirically distinguishing between these hypotheses and the hypothesis of no self-interaction. Similarly, Big Bang Nucleosynthesis constrains interactions between SIDM and 
baryons, and it constrains the potential coupling of the force carrier of the self-interaction to the Standard Model (Cyburt et al. 2002; Kouvaris et al. 2015).

The SIDM hypothesis has yielded striking results. When cosmological simulations include a self-interaction cross-section for dark matter particles, it seems that some of the small-scale challenges disappear (see Blennow et al. 2017). Some zoom-in simulations including SIDM show that both the discrepancies embodied in the Too Big To Fail and the Core/Cusp problems might never arise if SIDM is incorporated into the simulations (see for example Elbert et al. 2014 and 2018; Ren et al. 2019). Tulin and Yu (2017) recognize that the small-scale challenges "may signal the importance of baryon dynamics, or other limitations interpreting the observations" (69), but they nonetheless note that "the particle dynamics of self-interacting dynamics can provide a viable explanation" (ibid.).

Another example of the 'switch gears'-approach is warm dark matter (WDM). WDM candidates would be dark matter particles with non-negligible velocity distributions. One effect of WDM would be to wash out structure-seeding perturbations smaller than a certain scale set by the particular model under consideration. This has the potential to help with the Missing Satellites problem for instance, by preventing small satellite galaxies from forming in the first place. The main theoretical contenders are sterile neutrinos and gravitinos. To take just one example of current work in this area, Bozek et al. (2018) explore the effects of modeling DM as a resonantly-produced sterile neutrino (RPSN) on hydrodynamical dwarf galaxy simulations, which they compare to corresponding results produced with CDM. The choice to investigate an RPSN is at least in part motivated by a suggestive bit of X-ray data from some galaxy centers: a $3.55 \mathrm{keV}$ line of as yet 
unknown origin (cf. Boyarsky et al. 2014), although, Bozek et al. caution that "both the existence of the line and its interpretation remain controversial."

The initial success of these modifications to CDM appears encouraging, suggesting that 'switching gears' might be the recommended path forward to solve the small-scale challenges. We believe there is reason to be cautious, however. First, we have only mentioned a few possibilities for modifying the CDM hypothesis, but there are many more. All modifications come with their own partial success and failures (e.g. solving the Missing Satellites problem but not the Cusp/Core problem, or vice versa)—none are entirely successful. This makes it unclear what direction to pursue. Second, there is no solid independent empirical evidence supporting any of these modifications - their sole empirical motivation is resolving the small-scale challenges. For our purposes, mere theoretical possibility is not sufficiently "independent" motivation for solutions to the small-scale challenges. There are lots of possibilities in Beyond the Standard Model physics, and detection of such new physics (independent of the small-scale challenges themselves) is necessary for accepting such physics as causally responsible for the small-scale challenges. Choosing a modification and tuning its free parameters to resolve the small-scale challenges therefore seems to be nothing less than an ad hoc fix (we return to this line of thinking in Section 4 below).

\subsection{Keep on chugging: be patient and focus on advancing the simulations and their predictions}

In contrast to the previous two approaches, other researchers think that these apparent anomalies do not yet present insurmountable challenges to $\Lambda \mathrm{CDM}$. Rather, they believe that at least some of these challenges might reflect unwarranted assumptions made in matter- and gravitation-only simulations, problems with how empirical predictions are derived from the simulation outputs, overzealous interpretations of the observational data, or some combination of these. Before 
interpreting the small-scale challenges as genuine anomalies with respect to $\Lambda \mathrm{CDM}$, they propose elaborating on the details of the CDM hypothesis.

The small-scale challenges arise out of the application of matter and gravitation-only simulations in contexts where including additional physical processes is almost certainly important to adequately simulating structure formation at the relevant scales. Various efforts are underway to include additional non-gravitational physical processes in so-called hydrodynamical simulations. These simulations require much more computing power than gravitation-only simulations, and still require various simplifying assumptions. For present purposes, we will mention just two such projects that specialize in different scales: Illustris and Latte on FIRE.

Illustris is a project consisting of several large-scale hydrodynamical simulations (Genel et al. 2014; Rodriguez-Gomez et al. 2015; Sijacki et al. 2015; Torrey et al. 2014; Vogelsberger et al. 2014; Zhu et al. 2016). Illustris essentially models a standard $\Lambda$ CDM cosmology at large scales with a variety of astrophysical processes. For instance, Illustris-1 follows $1820^{3}$ dark matter "particles" representing $6.26 \times 10^{6}$ solar masses each, and approximately the same number of baryonic resolution elements (with average masses representing $1.26 \times 10^{6}$ solar masses) in a periodic box representing 106.5 Mpc on a side evolved to $\mathrm{z}=0$ with moving-mesh code AREPO (Genel et al. 2014, 177). In addition to evolving these particles under the influence of modeled gravity, hydrodynamics, and an ionizing background in an expanding universe, the simulation includes representations of radiative cooling, star formation, stellar evolution, star formation feedback with supernova explosions driving galactic winds, black hole seeding, accretion and merging, and active galactic nuclei feedback in three different modes (ibid.). Note that a standard six parameter $\Lambda$ CDM cosmology is assumed $\left(\Omega_{\Lambda}=0.7274, \Omega_{\text {matter }}=0.2726, \Omega_{\text {baryon }}=0.0456, \sigma_{8}=0.809, n_{\mathrm{s}}=0.963\right.$, and $H_{0}$ 
$\left.=70.4 \mathrm{~km} \mathrm{~s}^{-1} \mathrm{Mpc}^{-1}\right)^{6}$ The function of these simulations therefore, is really to see what happens in a $\Lambda \mathrm{CDM}$ cosmology on large scales, when simulations include more realistic physical processes in the evolution of the model than they have so far.

Other collaborations focus on smaller-scale, higher resolution simulations. Consider for instance, the Latte Project: the Milky Way on Feedback in Relativistic Environments (FIRE). As the name suggests, rather than simulating the universe on large scales, this project aims to simulate galaxies of masses similar to our own Milky Way along with their attending satellite dwarf galaxies (Wetzel et al. 2016). These simulations also assume $\Lambda \operatorname{CDM}\left(\Omega_{\Lambda}=0.728, \Omega_{\text {matter }}=0.272, \Omega_{\text {baryon }}=\right.$ $0.0455, h=0.702, \sigma_{8}=0.807$, and $\left.n_{s}=0.961\right)^{7}$, GIZMO code for modeling gravity, and the mesh-free finite-mass (MDM) method for hydrodynamical calculations and FIRE-2 for star formation and feedback. They include representations of radiative cooling and heating, atomic molecular, and metal-line cooling for select elements, an ionizing ultraviolet background, cosmic reionization, as well as a suite of stellar feedback processes including radiation pressure from massive stars, local photoionization and photoelectric heating, stellar winds, and both core collapse and Type Ia supernovae (Wetzel et al. 2016, 2). Unlike Illustris, this group uses a "zoom-in" approach, which means that they run a relatively large-scale dark-matter-only simulation first (a periodic box $85.5 \mathrm{Mpc}$ on a side), choose an isolated dark matter halo at $\mathrm{z}=0$ (the redshift corresponding to today) that meets certain specifications, trace particles within a bubble around that halo back to $z=100$ (i.e. earlier), and then re-simulate that area at higher resolution (ibid.) For

\footnotetext{
${ }^{6} \Omega_{\Lambda}, \Omega_{\text {matter }}$ and $\Omega_{\text {baryon }}$ are parameters expressing contributions to the total energy density of the universe from dark energy, total matter, and baryonic matter respectively, all proportional to the critical energy density at which the curvature of the universe is zero. The parameter $\mathrm{H}_{0}$ is the Hubble parameter today, $\sigma_{8}$ tracks a scale important for modeling the clustering of matter in the universe over cosmic history, and $n_{s}$, the "scalar spectral index", is a parameter that encodes information about possible gravitational influences in the early universe. For introductory technical definitions of cosmological parameters see Planck Collaboration (2013).

${ }^{7}$ See footnote 6 for explanations of the parameters. $\mathrm{h}$ is a dimensionless representation of $\mathrm{H}_{0}: \mathrm{h}=\mathrm{H}_{0} / 100 \mathrm{~s} * \mathrm{Mpc} / \mathrm{km}$.
} 
comparison with Illustris, their zoom-in region contains 140 million particles with each dark matter "particle" representing $3.5 \times 10^{4}$ solar masses. Intriguingly, these simulations do not display a Missing Satellites nor Too Big to Fail problem, which Wetzel et al. interpreted optimistically as meaning "that baryonic physics can account for these observations and thus reconcile dwarf galaxies with standard $\Lambda$ CDM cosmology" (ibid., 6).

Both approaches make advances in cosmological simulations and towards resolving the small-scale challenges by including representations of known physics, without modifying the CDM hypothesis. The fact that they have been to some extent successful is encouraging, and future projects are expected to build on this. Their respective successes are based on different approaches, which raises new questions: are the background assumptions of Illustris and Latte on FIRE equivalent, and how can we learn this? How can the epistemic impact of these assumptions be tested? We will return to these questions in Section 5.

Another aspect of elaborating the CDM hypothesis focuses on the methods used to derive empirical predictions from the gravitation-only simulations. Gravitation-only simulations result in a model representing the structure of dark matter halos only-they do not simulate how galaxies and clusters are formed within those halos. However, observational surveys do not map dark matter 'directly' but rather map galaxies and galaxy clusters via the electromagnetic spectrum (or infer dark matter distribution in the case of weak lensing surveys). Thus, to compare simulation outputs to the results of observational surveys, cosmologists are tasked with making reasonable assumptions about halo abundance matching and this has, as one might imagine, introduced substantial headaches related to populating dark matter halos with luminous galaxies ("galaxy bias", see Desjacques et al. 2018 for a review). This becomes relevant for at least one of the small-scale challenges; in fact, 
Bullock \& Boylan-Kolchin (2017) suggest that the Missing Satellites problem could be solved by "simply adapting the abundance-matching relation derived from field galaxies to 'solve' the missing satellites problem down to the scale of the classical Milky Way galaxies” (361).

This approach, chugging along, is the most optimistic of the three with respect to the possibility of resolving the small-scale challenges using the resources of $\Lambda \mathrm{CDM}$. Proponents of this approach hold off on introducing any genuinely novel physical theorizing, instead adding to the complexity of the existing models and re-checking the interpretation of the relevant empirical results. This approach comes with additional practical challenges relating to computing power and modeling many complicated phenomena together, but it does avoid introducing genuinely new physical theory about the universe and its components.

\section{Prioritizing patience}

How should physicists move forward in light of these small-scale challenges? Should they jump ship, abandoning the dark matter paradigm altogether, thereby abandoning a central part of the $\Lambda \mathrm{CDM}$ research programme as a whole? Should they shift gears, exploring possible modifications to our understanding of dark matter such as SIDM or WDM to see if those might accommodate the challenges? Or should they just keep on chugging, continuing to build cosmological simulations outfitted with more, and more detailed, physical processes?

While modification or even full-fledged replacement of the current cold dark matter paradigm may eventually be required, we argue that incorporating the effects of known physics should be prioritized at this time. In other words, we think that for now, modelers ought to just keep on chugging in response to the small-scale challenges. Cosmology simulations ought to include high fidelity representations of realistic astrophysical processes, including for instance gas cooling, 
photo-ionization, stellar evolution, and feedback from supernovae and black holes, and the small-scale challenges re-assessed, before appeal to new physics ought to be invoked in an attempt to address those challenges. We offer two main reasons in support of this position. In Section 5, we explore different scenarios and how the reasons offered here might apply to them.

\subsection{Epistemic conservatism}

The first reason to prefer including known physics is the general methodological heuristic we call epistemic conservatism: refraining from introducing new physics in the absence of compelling empirical evidence (cf. Worrall 2002). We believe epistemic conservatism in this sense is important in order to avoid making ad hoc modifications to a good theoretical model.

In the case of the small-scale challenges, our current best understanding of astrophysics implies that baryonic physics will have some effect on galactic and sub-galactic scales that is not yet accurately represented in predictions derived from cosmological simulations. Illustris and Latte on FIRE represent different attempts at including this baryonic physics. The fact that diverse approaches to including baryonic physics exist illustrates that no one knows precisely bow all of the ordinary physics affects the scales in question. Nonetheless, Illustris and Latte on FIRE have both been developed under the shared assumption that baryonic physics should affect (sub-)galactic scales_-an assumption justified by the simple fact that stellar winds, active galactic nuclei, and other feedback are energetic processes observed on such scales. In other words, there is evidence independent from the small-scale challenges in support of baryonic physics impacting structure formation at (sub-)galactic scales since there is independent empirical evidence for the existence of these physical processes and their powers to disrupt and otherwise alter structure formation. One 
goal of hydrodynamical simulations is to investigate what that impact is, and whether or not it might also have implications for the small-scale challenges.

The case for WDM or SIDM looks more bleak: the small-scale challenges currently are the only empirical motivation for these modifications to the CDM hypothesis. There is no independent empirical evidence for new physics playing a role on (sub-)galactic scales. Instead, all the weight is put on the small-scale challenges revealing new physics, regardless of whether or not baryonic physics plays a role on (sub-)galactic scales.

Granted, we also do not have good reason to believe that new physics will not have an effect on the small-scale challenges, nor is there evidence contradicting the possibility of dark matter being self-interacting or warm. Our claim with regards to modifications of dark matter is merely one about the absence of evidence, not about evidence to the contrary. ${ }^{8}$ This leaves open the possibility to investigate the effects of new physics for other reasons than just resolving the small-scale challenges. We come back to this possibility in the next section.

From the variety of approaches discussed in Section 3, it should be clear that the physics community is currently undecided as to what the small-scale challenges provide evidence for. Do the small-scale challenges provide evidence for additional effects of baryonic physics, or some new physics? Abandoning or modifying the CDM hypothesis amounts to interpreting the small-scale challenges as empirical support for new, non-baryonic physics. This interpretation implies that one assumes that baryonic physics cannot resolve them without having explored the full impact of baryonic physics on the simulation outputs. Thus, modifying the dark matter hypothesis is an

\footnotetext{
${ }^{8}$ The same is not true for MOND, as was discussed earlier.
} 
epistemically risky approach: it risks overstating conclusions based on a limited evidential basis and making potentially unnecessary ad hoc assumptions to accommodate the small-scale challenges.

At this point, advocates for MOND or modifications of the CDM hypothesis might attempt to charge us with inconsistent reasoning by noting that at an earlier point in history, CDM also constituted genuinely new physics. Following our reasoning applying epistemic conservatism, shouldn't dark matter itself have been judged as too risky?

The relevant difference between the historical contexts is that when dark matter was first introduced as a possible explanation for the flat galaxy rotation curves, it was already clear that no existing physical theory could explain the rotation curves. Both MOND and dark matter were considered somewhat plausible solutions at the time-the reason dark matter became the broadly accepted solution in the following decades is that it successfully explained a variety of newly observed phenomena on a wide range of scales, from cosmological structure formation to the Bullet Cluster. This success changed dark matter's status from a hypothetical explanation for galaxy rotation curves, to an essential component of the concordance model of cosmology. Today, in the context of the small-scale challenges, dark matter can therefore be considered 'standard physics'.

\subsection{Individuating contributing causal factors}

The second reason we offer in favor of prioritizing patience focuses on how contributing causal factors can be individuated. The current situation in dark matter research bears resemblance to a very common sort of situation in empirical science: researchers want to know which amongst, and to what extent, a variety of hypothesized causes contribute to some effect. If possible, it can be useful to try to study one contributing factor while holding the others fixed in hope of isolating the influence of that factor on the effect of interest. It is for this reason that experiments including 
control trials, or post-data collection analysis aiming to account for all potential sources of systematic error, can be so useful epistemically. In the context of astrophysics, Anderl (2018) offers a specific example: simple models with only a limited set of free parameters can sometimes help to uncover so-called "degeneracies", that is, different solutions in unconnected regions of parameter space that nonetheless all adequately recover observations due to various parameters being related to another. ' Similarly, Smeenk and Gallagher (Forthcoming, Section 5) argue that benchmarking exercises, increased modularity, and tracking down errors with eliminative reasoning can be useful for untangling the influence of interacting components in cosmological simulations and for avoiding mistaking spurious agreement between those simulations and empirical results as confirmatory.

In the case of dark matter, researchers want to know the extent to which and in what ways baryonic physics influences structure formation, and whether hypothesized modifications to our model of cosmology such as WDM or SIDM would also affect structure formation and if so, the extent to which and in what ways they would. However, including new physics like WDM or SIDM in simulations that are used to generate empirical predictions before ordinary baryonic physics has been satisfactorily included would be to unhelpfully "turn two knobs at once". ${ }^{10}$ In other words, it would be difficult for researchers to assess the extent to which adding realistic baryonic physics to our cosmological models will assuage the apparent anomalies embodied in the small-scale challenges,

\footnotetext{
${ }^{9}$ Anderl introduces a second use for simple models in astrophysics: assessing structural uncertainty. We will not discuss this in the current paper.

${ }^{10}$ While we believe that our analysis would be supplemented by a thorough explication using the resources of causal modeling, this task is beyond the scope of the present work. One way forward, for instance, would be to develop an account building upon Woodward's (2003) view. For Woodward, a variable " $\mathrm{X}$ is a contributing cause of $\mathrm{Y}$ with respect to [a set of variables] $\mathrm{V}$ if and only if there are changes in $\mathrm{X}$ that will change $\mathrm{Y}$ when the right other variables are held fixed at some value" (60). Applied to the current case, the suspicion is that baryonic physics is causally relevant to structure formation in a way that bears on the small-scale challenges, but this will be difficult to check without holding other variables fixed. Perhaps modifications to the dark matter hypothesis like SIDM and WDM will turn out to be actual contributing causes as well. To find out if this is so, we ought to be able to fix the "baryonic physics variable" at its actual value, which, whatever it is, will not be 0 .
} 
if other hypothetical contributors (with no independent evidentiary basis of their own) are being altered at the same time.

At this point, a proponent of introducing new physics might argue that while hydrodynamical simulations can focus on the contributing effects of baryonic physics within a CDM framework, there is no reason to refrain from exploring modifications to the cold dark matter paradigm in parallel. However, we believe that there are good reasons to not pursue the modifications to the CDM hypothesis in the current epistemic situation. First, pursuing SIDM or WDM in parallel assumes that we have a good understanding of how baryonic physics can be included as a background factor to be "held fixed". We do not. ${ }^{11}$ There is a wide range of hydrodynamical processes that might influence structure on (sub-)galactic scales, as our brief introduction into Illustris and FIRE above aimed to make clear. This means that it is as yet unknown what the "background conditions" for new physics variation would even consist in. Second, it is also not clear that there should be a self-interaction cross-section for dark matter or that dark matter should be warm. As we discussed above, these are hypotheses with no solid independent empirical evidence to support them yet. Incorporating such new physics into cosmological simulations thus runs the risk of introducing spurious causes.

Finally, while the first two points complicate the determination of the (potential) causes, there is also a possible problem on the side of the effect. The Missing Satellites problem and Too Big to Fail problem both arose based on observations of the Milky Way, and, to some extent, the Andromeda

\footnotetext{
${ }^{11}$ An anonymous reviewer suggested that we consider the possibility that researchers could succeed in "turning two knobs at once" in the context of the small-scale changes in light of apparent successes in simultaneously modeling multiple contributing causes that are jointly responsible for some phenomenon in other areas of physics. To clarify: we do not deny the possibility of simultaneously modeling multiple contributing causal factors. Rather, we argue that doing so successfully requires enough independent justification for the various contributing causes to avoid introducing degrees of freedom that would be shielded from empirical constraint.
} 
galaxy. It is possible that the Milky Way is not a typical galaxy, and that those two small-scale challenges do not arise in general. This has been the focus of the SAGA Survey (Geha et al. 2017, see also Kim et al. 2018). Suppose including new physics like SIDM or WDM "solved" the small-scale challenges. This "success" could produce the following embarrassing scenario. If it turns out that the Missing Satellites and Too Big To Fail problems are not general problems relevant on the scale of the cosmic arena but rather reflect particularities in our local group of galaxies, then by incorporating new physics, modelers would have (unnecessarily) succeeded in accounting for a fictitious effect. The prospect of this sort of embarrassment does not arise in the case of baryonic physics because modelers have good empirical reasons for thinking that astrophysical processes like supernovae and active galactic nuclei are present and have some effect on galaxy formation broadly, regardless of the small-scale challenges. Even if the small-scale challenges turn out not to be challenges after all, it would still be worthwhile to simulate the effects of realistic astrophysical processes on structure formation.

To summarize, if new physics is incorporated before or simultaneously with the effects of ordinary physics, the effects of new physics could mask or overcompensate for effects that should have been attributed to ordinary physics. Modelers should not "turn both knobs" at once - they should settle on the effects of the ordinary first and then see if anomalies remain.

\section{Heuristics applied to future scenarios}

In Section 4, we introduced two reasons to prioritize incorporating known physics in simulations in context of the small-scale challenges: 1) there is as yet no independent empirical motivation for switching gears by modifying the dark matter hypothesis, thereby rendering such modifications ad hoc and 2) switching gears before known physics has been satisfactorily included runs the risk of 
muddying causal understanding and "solving" spurious challenges. To see these reasons at work, we now consider different possible scenarios for future research in dark matter and we discuss what our recommendation would be in each case. For clarity, when we refer to "hydrodynamical simulations" or "simulations" in this context, we mean simulations that incorporate baryonic physics, without modifying the cold dark matter-hypothesis.

Scenario 1, Univocal success: The most ideal scenario is one where one hydrodynamical simulation with well-supported modeling assumptions succeeds in resolving all small-scale challenges, while other approaches fail to resolve in some way (e.g. lack of support for assumptions or failure to yield solutions). Given the current status of the field and the complexity of hydrodynamical simulations, this scenario is not very likely.

Why is this scenario ideal? First, it shows that the epistemic conservatism was justified. There is no independent evidence to introduce new physics-baryonic physics accommodates the small-scale challenges, which therefore do not constitute compelling evidence for the introduction of new physics. Moreover, since no new physics was introduced, there is also no risk in new physics causing confusion in the causal relations. It is plausible all contributing causes are included in the baryonic physics, since there is no residual effect to be accounted for. Physicists can keep working on the successful simulation, and use similarities and differences with the unsuccessful ones to refine understanding of the influence of baryonic physics and auxiliary simulation assumptions.

We mention "well-supported modeling assumptions" with recent concerns about numerical effects in cosmological simulations masquerading as physically significant predictions in mind. For 
instance, theoretical astrophysicist Frank van den Bosch and his research group have argued that numerical effects can (and have) disrupted modeled structure formation in state-of-the-art cosmological simulations (van den Bosch et al. 2018; van den Bosch \& Ogiya 2018). Analyzing this research with reference to the robustness literature in philosophy of science, Gueguen (Forthcoming) argues that convergence of cosmological simulation results "is not sufficient to exclude numerical artifacts and that robust predictions cannot be considered reliable on such ground" in this context, and that furthermore, there is reason to think that apparent convergence of simulations in the case of the Cusp/Core problem actually results from numerical artifacts. In light of these findings, we take it that a necessary condition for $\Lambda \mathrm{CDM}$ achieving univocal success in resolving the small-scale challenges is good understanding and control over such numerical effects in the simulations.

Scenario 1 explicates our standards for successful resolution of the small-scale challenges. We would consider them to be "resolved" in an adequate manner if:

1. The hydrodynamical simulation in question is unproblematic given our background knowledge, for example with regards to how the baryonic physics has been included and how predictions have been derived from it. Currently, no hydrodynamic simulation satisfies this condition;

2. The predictions of the simulation match the observations within the standards of the discipline;

3. No argument has been constructed that some relevant physics has not been accounted for, given our background knowledge. 
If these conditions are fulfilled, we believe the small-scale challenges would effectively have disappeared. $^{12}$ On such an occasion, it might be worthwhile to continue research into specific causal pathways to see what specific baryonic physics explains what specific process of structure formation, but that research could no longer count against the CDM framework. It would rather be a project in refining our understanding of the CDM framework and the effects of baryonic physics.

Scenario 2, Apparent underdetermination: Different hydrodynamical simulations each resolve all small-scale challenges, but their physical model of the influence of baryonic physics, as well as its computational implementation differ and each variant enjoys apparently equally well-supported modeling assumptions.

Similar to the first scenario, this scenario shows that epistemic conservatism is once again vindicated: the small-scale challenges do not provide compelling evidence for new physics. The main concern here is individuation of the contributing causal factors. It might be the case that all contributing causes have been identified as baryonic in at least one of the hydrodynamical simulations, but more work needs to be done to understand why simulations with different physical models or computational implementations still all succeed in resolving the small-scale challenges. ${ }^{13}$

\footnotetext{
${ }^{12}$ Massimi (2018) seems to take a different stance. She casts doubt on the ability of cosmological simulations with realistic baryonic physics to provide satisfactory causal explanations of precise astrophysical data and likens the production of empirically adequate simulation outputs with "curve fitting" (34). If we understand Massimi's point here correctly, we may be more optimistic than she that cosmologists can come to know enough about the contributions that the interacting components of their models make in order to understand how these components contribute to the outputs of their simulations in ways significantly more satisfying than curve fitting. In particular, securing a physically plausible narrative about the causal contributions seems worth striving for, even if tracking causal interactions step-by-step in these complicated simulations would be both too big of a challenge and unenlightening.

${ }^{13}$ One might be tempted to invoke pragmatic values like simplicit and explanatory power to break the apparent underdetermination exhibited in this scenario. We thank an anonymous referee for pressing this point. However, doing so would be ill-advised in the long term since it would curtail further efforts to uncover subtle differences in the models whose empirical implications might be further teased out and differentiated.
} 
One possibility is that the models and implementations overlap in crucial areas that are responsible for the resolution of the small-scale challenges; another is that the different simulations each identify different processes that are all potentially responsible for the resolution of the small-scale challenges; yet another possibility is that some of the successful simulations suffer from spurious artefacts. Exploring similarities and differences between successful simulations can move research forward.

Scenario 3, Patchy success: Different simulations each resolve some of the small-scale challenges, but no single set of simulations implementing one consistent overarching model resolves all of them.

Given the success of Illustris and Latte on FIRE, we consider this the current situation in the field. There is not enough reason just yet to give up on epistemic conservatism, although it is also not clear that conservatism will be vindicated. Similarly, it is at least plausible that some contributing causes have been identified, but more work is required to understand why simulations with different background assumptions manage to resolve some but not all of the challenges. Understanding the baryonic physics is also a prerequisite to determine what residual effects remain after all baryonic physics has been included. As mentioned above, it will also be interesting to see if some of the small-scale challenges disappear independently of improvements aimed at making simulations more realistic, for instance by turning out to reflect peculiarities about our own galaxy.

Scenario 4, Persistent anomalies: In a less optimistic scenario, it could be the case that no well-motivated hydrodynamical simulation satisfactorily solves any of the small-scale challenges within the current technological limitations, but that current background knowledge makes it probable that including 
more baryonic physics would make a difference in the resolution of the small-scale challenges, were it technologically possible. ${ }^{14}$

Epistemic conservatism is still a useful heuristic in this scenario: the small-scale challenges do not provide conclusive evidence for the introduction of new physics, but it is also not quite clear that the challenges do not provide some evidence for new physics after all, given our background knowledge. Similarly, with regards to individuating contributing causes, it is not clear yet whether baryonic physics alone can resolve the small-scale challenges, or whether there would be residual discrepancies between the predictions and the empirical results if the baryonic physics was satisfactorily represented.

At this point, we believe it would be rationally permissible for pragmatic reasons to start exploring the effects of adding new physics insofar as refusing to do so would amount to a refusal to do more research into the small-scale challenges. However, awareness that the effects of baryonic physics have not been adequately accounted for just yet ought to be retained. If in this scenario, the addition of new physics solved all small-scale challenges, that would actually provide good reason to not take the new physics at face value: there would be good reasons to believe that the new physics would be overcompensating for effects that should instead be attributed to ordinary physics. Without extending technological limits, it would remain impossible to know exactly which effects this concern applies to, of course.

\footnotetext{
14 This as well as the next scenario are less realistic, given the current state of the field. They are nonetheless useful to consider to elaborate on the reasons discussed in Section 4.
} 
Scenario 5, Empirical inadequacy: It could also be the case that no hydrodynamical simulation satisfactorily solves any of the small-scale challenges within current technological limitations, but that background knowledge makes it plausible that including more baryonic physics would not make a difference.

This we consider a scenario in which modifying the cold dark matter hypothesis would be warranted. In this scenario, the small-scale challenges ought to be interpreted as genuine anomalies for $\Lambda \mathrm{CDM}$; all resolutions within the framework of $\Lambda \mathrm{CDM}$ using established baryonic physics have been excluded. It therefore becomes reasonable to give up on the heuristic of epistemic conservatism.

Similarly, in such circumstances the worries about new physics overcompensating for potential causal effects of baryonic physics no longer apply. Baryonic physics alone did not suffice to accommodate the small-scale challenges, and new physics is required to account for the residual effect. The only available option at this point is beginning to incorporate new physics-hopefully with some independent evidence as to how to proceed. ${ }^{15}$

Finally, let us briefly touch upon the possibility that new evidence on the particle nature of dark matter is discovered, for example from direct detection or production experiments. In general, this would be good reason to include this specific new physics in the simulations, insofar as the evidence warrants. The new evidence does not remove the need to also further incorporate baryonic physics

\footnotetext{
${ }^{15}$ In this case, both modifications to the dark matter particle properties, as well as the 'ugly' solution of cold dark matter with some modified gravity could be pursued. The latter has previously been discussed in a philosophical exchange about the Bullet Cluster (see Kosso 2013, Sus 2014, Vanderburgh 2014). While the Bullet Cluster observations can be a useful resource to constrain such gear-shifting approaches, these constraints remain useless unless all relevant baryonic physics has been accounted for. We thank an anonymous referee for pointing us towards these papers.
} 
in hydrodynamical simulations, however, unless there is independent reason to forego this (as discussed in Scenario 5).

\section{Pluralists' objections}

Our argument suggests prioritizing one methodological strategy over others, and therefore amounts to a mild version of methodological monism. ${ }^{16}$ Two nuances are in order here. First, this monism is based purely on epistemic appraisal. In other words, our focus here is on maximizing the likelihood of the prioritized theoretical model being epistemically superior to competitors, as well as the likelihood of scientists being able to determine that that model is epistemically superior. Of course, we are aware that there can also be pragmatic reasons for pursuit (as we discussed in Section 5). The implications of our argument for scientific practice will thus depend on how epistemic and pragmatic reasons are balanced. Nonetheless, if one accepts our epistemic analysis of the current debate, we believe our arguments should give reason to oppose a blanket methodological pluralism without any type of prioritization.

Second, it may be particular to this debate that the various available responses to the small-scale challenges neatly fall into the three categories outlined in Section 3. The terrain of other debates may be different Similarly, it may be particular to this debate that the two heuristics we introduced both point to one out of three strategies. Thus, while the two heuristics clearly favor prioritizing one type of strategy over all others here, we do not claim that that will be the case in all other contexts where they apply.

Yet a proponent of methodological pluralism may object that humility disfavors the sort of prioritization of strategies for which we have argued and instead recommends permissive methodological pluralism (we thank our referees for pressing this point). In general, preference for

\footnotetext{
${ }^{16}$ We thank an anonymous referee for encouraging us to clarify this point.
} 
different virtues of the sort found in Kuhn's (1977) well-known list (accuracy, consistency, breadth of scope, simplicity, and fruitfulness) can lead scientists to judge different research programmes as more or less pursuit-worthy. In particular, one might think that diverse preferences with respect to such virtues could support rational disagreement, or what Bradley (2017) calls "rational silence", regarding which approaches are deemed pursuit-worthy. In the context of the small-scale challenges, epistemic humility may recommend tolerating MONDian ship-jumping for those researchers who happen to lean heavily on theoretical virtues such as explanatory power.

In response to this objection, we want to stress that our argument does not rule out the appropriateness of methodological pluralism resulting from different virtue preferences in general. Scientists may often quite reasonably choose to pursue different research programmes on diverse pragmatic or cognitive grounds. Instead, our position is that in the case of the small-scale challenges, the nature of the epistemic situation makes shifting gears before including known physics a dead end. For this not to be the case, it would have to turn out that baryonic physics was not relevant to the formation and evolution of structure at the scale of galaxies and galaxy clusters. We are certainly not claiming that researchers should be banned from investigating alternatives to $\Lambda \mathrm{CDM}$. Nor are we claiming that such research is not worthy of material support e.g. in the way of funding, credit incentives, and the like. Rather, our claim is that any researchers choosing to pursue gear-shifting approaches like WDM and SIDM will eventually have to contend with the relevance of ordinary baryonic physics to the structure and evolution of galaxies and galaxy clusters. Ship-jumpers will eventually also have to contend with recovering the empirical successes of $\Lambda \mathrm{CDM}$ at cosmic scales. These, we have argued, are reasons to prioritize including baryonic physics in an attempt to solve the small-scale challenges, which transcend diverse preferences for pragmatic or cognitive virtues that 
might otherwise play a role in the adjudication of pursuit-worthiness with respect to research programmes.

Still, one might question whether our argument ignores the utility of maintaining, or perhaps even incentivising, some risky exploration in science. Isn't it the quixotic figures, those who throw conservatism to the wind and take the road less traveled, that end up being the revolutionaries and bring about the progressive changes in our scientific worldview?

Recent work in social epistemology and philosophy of science exploring these questions with formal modeling tools has yielded suggestive results. While we cannot hope to address that entire literature here, we present and respond to a plausible objection on behalf of an instance of particularly relevant and striking research in this genre. Following on research by Zollman (2010), Kummerfeld and Zollman (2016) demonstrate that, under particular modeling assumptions, scientists facing a choice between pursuing a conservative research programme and an exploratory one will collectively act inefficiently-they will tend to be too conservative. Kummerfeld and Zollman conclude "that governments and foundations should reverse the general trend and intervene to encourage risky science beyond what would occur without their intervention" (1059).

The sort of result presented by Kummerfeld and Zollman poses a prima facie challenge to the cogency of our argument. Invoking conservatism, we advocate for prioritizing patience in responding to the small-scale challenges, for chugging along by working to incorporate known physics into simulations. If Kummerfeld and Zollman are right, there may be reason to encourage risker alternatives by, say, incentivising research in MOND.

Ultimately, we believe this implication for our case lacks justification. To explain why, it will be helpful to provide slightly more detail regarding Kummerfeld and Zollman's model. These 
authors argue that scientists in a scientific community left to its own devices, that is, without institutional interventions such as grants and prizes, often face decisions that can be modeled as "bandit problems". A bandit problem (named after "one arm bandits"-slot machines) is a decision-theoretic scenario in which an agent faces an iterative choice among options with unknown payoffs, like which slot machine to play in a casino room. Should the agent continue to play the machine that, given present knowledge, is expected to give the highest payout? Or should the agent take a risk and try other machines in the hope that they may pay more? A simple example would be a case in which an agent iteratively decides to pull one of two slot machine arms, the "safe choice" that always returns a payoff of $\$ 1$ and the "risky choice", which returns $\$ 3$ half of the time and $\$ 0$ otherwise (ibid., 1061-1064). The willingness of an agent to explore alternatives can be represented as her propensity to pull the risky choice arm after she has learned from experience that the safe choice arm regularly delivers $\$ 1$. Kummerfeld and Zollman appeal to discovery of the cause of peptic ulcers as a relevant example from the history of science (ibid., 1060-1061). The widespread view in the medical community used to be that the likely cause of ulcers was stomach acid and thus most of the research operated within a research programme premised on that assumption. A couple rogue scientists bucked the trend to explore the risker bacteria-based hypothesis, ultimately succeeding. Kummerfeld and Zollman show that if we consider a community of decision-makers confronted with the same choices, who are initially prone to exploration to a variety of degrees, and who can communicate among one another, the community will operate sub-optimally in that the individuals in the community will make decisions that, while appearing best at the individual level, result in the community doing worse that it would have done with more exploration of risky choices. 
${ }^{17}$ In particular, individuals will try to free-ride on the exploration of others, ultimately adversely affecting the performance of the community.

Setting aside several other concerns that ought to be addressed in applying Kummerfeld and Zollman's results to actual scientific communities facing decisions of the sort raised in the context of the small-scale challenges, ${ }^{18}$ there is a particularly important aspect of the small-scale challenges case that makes it a poor fit for modeling as a bandit problem. What would the "arms" be? To simplify, consider the choice that a certain researcher faces between pursuing MOND and chugging along with baryonic physics in the $\Lambda \mathrm{CDM}$ framework. Perhaps Kummerfeld and Zollman would want to model the MOND choice as "risky" and the $\Lambda$ CDM choice as "safe". Yet, it is important to note that in Kummerfeld and Zollman's models, the "risky" choice is set to be the right choice (ibid., 1075). In reality of course, this may not be the case-the research programme that scientists currently expect to deliver the most epistemic success could turn out to be superior to other alternatives it is possible to explore. Making the risky choice superior is a substantial modeling

\footnotetext{
${ }^{17}$ Heesen (2019) defines "maverick" scientists/“impact seekers", as opposed to "followers"/"safety seekers", as those who "go in for research in relatively unexplored areas, or areas where the foundations are not as settled" (5). That is, a researcher "who works on high-risk high-reward projects" (ibid., 6). The mavericks are those risk-taking scientists who will not settle for mere normal-science puzzle-solving, in the Kuhnian sense. While our argument does concern the mavericks vs. followers in this sense, unlike Heesen, we are not concerned with effective means of influencing the social structure of science so as to optimize the balance of scientists acting in these modes (Heesen argues that credit incentives like prizes may not be as effective as influencing tools as one might have expected). Rather, we are interested in the issue from the point of view of the scientists themselves: should I be a maverick or a follower regarding solutions to the small-scale challenges. Heesen claims that whether an individual scientist chooses to be a maverick or a follower is a matter of predisposition (ibid., 6). While we agree that maverick vs. follower tendencies may be significantly influenced by a scientist's personality, which may even be unlikely to change, we maintain the decision-making process of many scientists, regardless of personality, is susceptible to the arguments presented to them, such as that we offer in this paper. ${ }^{18}$ Some limitations of the real-world applicability of the model are explicitly discussed by the authors, for instance, the possibility that some scientists may not be perfectly self-interested and may have an iota of altruism regarding the epistemic advances of the scientific community as a whole (1074). The authors also rightfully emphasize that their model applies specifically to the scientific "state of nature" in which there are no institutional interventions-no Nobel Prizes - which is not our actual world (ibid.). In addition, it would be useful to consider how to correct their model using empirically-supported "behavioral" decision-making characteristics of actual humans and to further examine potential difficulties in applying evolutionary game theory to the dynamic realm of scientific institutions and social groups (see O'Connor 2019 for more work in this direction, and for qualificatory remarks about the utility of such models).
} 
assumption because Kummerfeld and Zollman also assume that the agents in their models are 'risk neutral'-they neither seek nor avoid risk for its own sake. They are careful to clarify that if actual scientists are risk seeking "to such an extent that they would seek out theories that are worse but risker", then the results of their modeling may not apply (ibid.). In other words, Kummerfeld and Zollman's results do not unconditionally support incentivising the exploration of risky options.

In our case, there really is no avoiding the need to include further known physics in predictive simulations of galaxy and galaxy-scale phenomena. In other words, what one might be tempted to model as the "safe" option infiltrates both bandit arms. There is not really a tenable choice between MOND on one hand and adding baryonic physics to $\Lambda$ CDM-based simulations on the other. The baryonic physics will be needed either way. Insofar as we represent this epistemic situation as involving a choice to pursue MOND without including the needed baryonic physics, then an agent who pursues that option ends up looking like a risk-seeker in the above sense: she chooses that arm in virtue of it being risky, yet it is worse. In that case, Kummerfeld and Zollman tell us that their results may no longer hold.

We therefore suggest that whether similar model-based results to those achieved by Kummerfeld and Zollman apply to our case warrants further investigation. Perhaps for instance, the choice between future-MOND and future- $\Lambda \mathrm{CDM}$, baryonic physics included, could be modeled as a bandit problem and could imply the epistemic desirability of incentivising more exploration of the risky option than would be expected were scientists to be left to their own devices. We grant that this possibility might be interesting to consider in its own appropriate context, given the state of evidence at that time. To be clear: we are not arguing against the further development of MOND (or SIDM, or WDM) in toto. MOND (or SIDM, or WDM) should not be preferred to $\Lambda \mathrm{CDM}$ as a 
solution to the apparent anomalies reflected in the small-scale challenges for the reasons we have articulated. This does not preclude the pursuit of alternative research programmes like MOND for their own sake. Whether a researcher chooses to work within the $\Lambda \mathrm{CDM}$ programme or explore and develop alternatives may largely be a matter of temperament and personal valuation of the variety of cognitive virtues that may be associated with different approaches. There may be further arguments to be made at the level of prioritizing and weighing virtues, which we do not pursue here. ${ }^{19}$ What does matter for our argument is that with regard to the apparent anomalies reflected in the small-scale challenges, there are good programme-allegiance-neutral reasons to prioritize patience insofar as one's aim is to develop the epistemically superior option. It is not currently reasonable for anyone to adopt MOND, SIDM, or WDM as a "solution" to the small-scale challenges.

\section{Conclusion}

We introduced the small-scale challenge as a live case manifesting unresolved normative questions about the methodology of theory change. We considered various responses: jumping ship entirely, shifting gears, or chugging along, and we have argued for the third option in this context. We conclude that patiently simulating known physics should be prioritized until either 1) practical limitations are reached (Scenario 4), or 2) all known physics has been incorporated to the extent that can be reasonably expected to make a difference (Scenario 5). For 1, we stress that incorporating known physics should remain an aim. We do not mean to suggest that adding in further realistic details to models is always the right thing to do. In particular, the arguments offered here do not speak against the value of certain minimal models, simplified models, and fictions in some scientific contexts and for some scientific purposes as articulated in the philosophy of science literature on

\footnotetext{
${ }^{19}$ For instance, Douglas (2013) quite rightfully points out that the virtues of empirical adequacy and internal consistency are necessary conditions of an acceptable scientific theory (799).
} 
modeling and idealization (see e.g. Batterman \& Rice 2014; McCoy \& Massimi 2018; Anderl 2018; Suárez 2010). Rather, the position we have aimed to defend is the local one: with respect to the present small-scale challenges in cosmology, there happen to be good reasons to add more realistic details into the models at this juncture.

Although we focused here on the small-scale challenges, we have drawn upon the heuristics of epistemic conservatism and isolating causal factors, which apply much more broadly. Epistemic conservatism has a venerable history in philosophy of scientific methodologies: it functions as the brakes preventing ad hoc modifications of a theory and the unwieldy proliferation of theories. The heuristic of isolating causal factors is also a familiar tool, if perhaps less obviously so. It is the lever that allows scientists to pry open causal systems to understand their machinations. When causal contributions are muddied or obscured, our confidence that we have understood what components of our models are responsible for what features of the phenomenon of interest is compromised (cf. Lenhard \& Winsberg 2010). In contrast, when we have a solid independent handle on the pieces of a modeling puzzle then we can be more confident in their composite representation. By invoking these heuristics in the particular context of the small-scale challenges we have demonstrated that they can be used contemporaneously to justify normative arguments in the midst of an unfolding debate about theory choice in which the possibility of impending revolution has surfaced.

Our cosmological case is one where it might appear that anomalies are accumulating, which might be thought to spell trouble for a reigning paradigm. The $\Lambda \mathrm{CDM}$ research programme might appear to be flagging in its capacity to address new problems. However, the concordance model of cosmology only appears to be in trouble with respect to the small-scale challenges, if you forget about the potential that this model already has for addressing the challenges. Ordinary baryonic 
physics, if given the chance to demonstrate its effects on structure formation, may well ameliorate the small-scale challenges. Simulation has to catch up to the riches already housed in the model.

\section{Bibliography}

Anderl, S. (2018). Simplicity and Simplification in Astrophysical Modeling. Philosophy of Science, 85(5): 819-831

Batterman, R. W. \& Rice, C. C. (2014). Minimal Model Explanations. Philosophy of Science 81(3): 349-376.

Blennow, M., Clementz, S., \& Herrero-Garcia, J. (2017). Self-interacting inelastic dark matter: A viable solution to the small scale structure problems. Journal of Cosmology and Astroparticle Physics, 2017(03): 048.

Boran, S. et al. (2018). GW170817 Falsifies Dark Matter Emulators. Physical Review D 97:041501(R).

Boyarsky, A., Ruchayskiy, O., Iakubovskyi, D. \& Franse, J. (2014). Unidentified line in X-ray spectra of the Andromeda galaxy and Perseus galaxy cluster. Physical Review Letters, 113(25): 251301

Bozek, B., Fitts, A., Boylan-Kolchin, M., Garrison-Kimmel, S., Abazajian, K., Bullock, J. S., ... \& Hopkins, P. F. (2018). Warm FIRE: simulating galaxy formation with resonant sterile neutrino dark matter. Monthly Notices of the Royal Astronomical Society, 483(3): 4086-4099.

Bradley, S. (2017). Constraints on Rational Theory Choice. The British Journal for Philosophy of Science 10(1): 1-24.

Bullock, J. S. \& Boylan-Kolchin, M. (2017). Small Scale Challenges to the $\Lambda$ CDM Paradigm. Annual Review of Astronomy and Astrophysics, 55(1)

Clowe, D., Bradac, M., Gonzalez, A. H., Markevitch, M., Randall, S. W., Jones, C. \& Zaritsky, D. 
(2006). A direct empirical proof of the existence of dark matter. The Astrophysical Journal, 648:

L109-L113.

Chall, C. (2020). Model-Groups as Scientific Research Programmes. European Journal for

Philosophy of Science 10(1): 1-24.

Cyburt, R. H., Fields, B. D., Pavlidou, V., \& Wandelt, B. (2002). Constraining strong baryon-dark-matter interactions with primordial nucleosynthesis and cosmic rays. Physical Review $D, 65(12)$.

Darden, L. (1991). Theory Change in Science: Strategies from Mendelian Genetics. Oxford: Oxford University Press.

Daston, L. \& P. Galison. (2007). Objectivity. Brooklyn, NY: Zone Books.

Desjacques, V., Jeong, D., \& Schmidt, F. (2018). Large-scale galaxy bias. Physics Reports, 733: 1-193.

Douglas, H. (2009). Science, Policy, and the Value-Free Ideal. University of Pittsburgh Press.

--- (2013). The Value of Cognitive Values. Philosophy of Science 80(5): 796-806.

Elbert, O. D., Bullock, J. S., Kaplinghat, M., Garrison-Kimmel, S., Graus, A. S. \& Rocha, M. (2018). A testable conspiracy: simulating baryonic effects on self-interacting dark matter halos. The Astrophysical Journal, 853(2): 109.

Elbert, O., Bullock, J. S., Garrison-Kimmel, S., Rocha, M., Oñorbe, J. \& Peter, A. H. G. (2014). Core Formation in Dwarf Halos with Self Interacting Dark Matter: No Fine-Tuning Necessary. Monthly Notices of the Royal Astronomical Society, 9(December): 9.

Famaey, B. \& McGaugh, S. (2012). Modified Newtonian Dynamics (MOND): Observational Phenomenology and Relativistic Extensions. Living Reviews in Relativity, 15(10).

Franklin, A. (2013). Shifting Standards: Experiments in Particle Physics in the Twentieth Century. University 
of Pittsburgh Press.

Geha, M., Wechsler, R. H., Mao, Y. Y., Tollerud, E. J., Weiner, B., Bernstein, R., ... \& Lu, Y. (2017). The SAGA Survey. I. Satellite Galaxy Populations around Eight Milky Way Analogs. The Astrophysical Journal, 847(1): 4.

Genel, S., Vogelsberger, M., Springel, V., Sijacki, D., Nelson, D., Snyder, G., ... \& Hernquist, L. (2014). Introducing the Illustris project: The evolution of galaxy populations across cosmic time. Monthly Notices of the Royal Astronomical Society, 445(1): 175-200.

Gueguen, M. (Forthcoming). On Robustness in Cosmological Simulations. Philosophy of Science.

Hacking, I. (Ed.). (1981). Scientific Revolutions. Oxford: Oxford University Press.

Heesen, R. (2019). The credit incentive to be a maverick. Studies in History and Philosophy of Science 76: 5-12.

Jeffrey, R. C. (1956). Valuation and Acceptance of Scientific Hypotheses. Philosophy of Science 23(3):237-246.

Kim, S. Y., A. H. G. Peter \& J. R. Hargis. (2018). Missing Satellites Problem: Completeness Corrections to the Number of Satellite Galaxies in the Milky Way are Consistent with Cold Dark Matter Predictions. Physical Review Letters 121: 211302.

Kosso, P. (2013). Evidence of dark matter, and the interpretive role of general relativity. Studies in History and Philosophy of Modern Physics 44(2): 143-147.

Kouvaris, C., Shoemaker, I. M. \& Tuominen, K. (2015). Self-interacting dark matter through the Higgs portal. Physical Review D, 91(4): 1-7.

Kuhn, T. S. (1962). The Structure of Scientific Revolutions. Chicago: University of Chicago Press.

--- (1977). “Objectivity, Value Judgment, and Theory Choice.” In The Essential Tension: Selected Studies 
in Scientific Tradition and Change, 320-39. Chicago: University of Chicago Press.

Kummerfeld, E. \& K. J. S. Zollman. (2016). Conservatism and the Scientific State of Nature. The British Journal for the Philosophy of Science 67(4): 1057-1076.

Lakatos, I. (1978). The Methodology of Scientific Research Programmes. Cambridge University Press.

Lenhard, J., \& Winsberg, E. (2010). Holism, entrenchment, and the future of climate model pluralism. Studies in History and Philosophy of Modern Physics, 41(3): 253-262.

Laudan, L. (1977). Progress and Its Problems. Berkeley, CA: University of California Press.

Longino, H. E. (1990). Science as Social Knowledge: V alues and Objectivity in Scientific Inquiry. Princeton: Princeton University Press.

Massimi, M. (2018). Three problems about multi-scale modelling in cosmology. Studies in History and Philosophy of Modern Physics 64: 26-38.

Milgrom, M. (1983). A Modification of the Newtonian Dynamics: Implications for Galaxies. The Astrophysical Journal, 270: 371-383.

McCoy, C. \& Massimi, M. (2018). Simplified Models: A Different Perspective on Models as Mediators. European Journal for Philosophy of Science 8(1): 99-123.

McGaugh, S. (2014). A tale of two paradigms: the mutual incommensurability of $\Lambda$ CDM and MOND. Canadian Journal of Physics, 93(2): 250-259.

O'Connor, C. (2019). The natural selection of conservative science. Studies in History and Philosophy of Science 76: 24-29.

Peacock, John A. (2015). Cosmological Physics. Cambridge University Press.

Planck Collaboration. (2013). Planck 2013 results. XVI. Cosmological parameters. Astronomy \& Astrophysics, 571: A16. 
--- (2018). Planck 2018 results. VI. Cosmological parameters. https://arxiv.org/abs/1807.06209

Ren, T., Kwa, A., Kaplinghat, M. \& Yu, H. B. (2019). Reconciling the diversity and uniformity of galactic rotation curves with self-interacting dark matter. Physical Review X, 9(3): 031020.

Rodriguez-Gomez, V., Genel, S., Vogelsberger, M., Sijacki, D., Pillepich, A., Sales, L. V., ... \& Hernquist, L. (2015). The merger rate of galaxies in the Illustris simulation: A comparison with observations and semi-empirical models. Monthly Notices of the Royal Astronomical Society, 449(1).

Rubin, V. C. \& Ford, W. K. J. (1970). Rotation of the Andromeda Nebula from a Spectroscopic Survey of Emission Regions. Astrophysical Journal, 159: 379.

Rudner, R. (1953). The Scientist Qua Scientist Makes Value Judgments. Philosopby of Science 20(1):1-6.

Sijacki, D., Vogelsberger, M., Genel, S., Springel, V., Torrey, P., Snyder, G. F., .. \& Hernquist, L. (2015). The Illustris simulation: The evolving population of black holes across cosmic time. Monthly Notices of the Royal Astronomical Society, 452(1): 575-596.

Smeenk, C. \& S. C. Gallagher (Forthcoming). Validating the Universe in a Box. Pbilosophy of Science

Spergel, D. N. \& Steinhardt, P. J. (2000). Observational Evidence for Self-Interacting Cold Dark Matter. Physical Review Letters, 84(17): 3760-3763.

Springel, V., White, S. D. M., Jenkins, A., Frenk, C. S., Yoshida, N., Gao, L., .. \& Pearce, F. (2005). Simulating the joint evolution of quasars, galaxies and their large-scale distribution. Nature 435: 629-636.

Suárez, M. (Ed.) (2010). Fictions in Science: Philosophical Essays on Modeling and Idealization. Routledge.

Sus, A. (2014). Dark matter, the Equivalence Principle, and modified gravity. Studies in History and Philosophy of Modern Physics, 45: 66-71. 
Torrey, P., Vogelsberger, M., Genel, S., Sijacki, D., Springel, V. \& Hernquist, L. (2014). A model for cosmological simulations of galaxy formation physics: Multi-epoch validation. Monthly Notices of the Royal Astronomical Society, 438(3): 1985-2004.

Thagard, P. (1993). Conceptual Revolutions. Princeton University Press.

Toulmin, S. E. (1972). Human Understanding, vol. 1: The Collective Use and Evolution of Concepts. Oxford: Clarendon Press.

Tulin, S. \& Yu, H.-B. (2017). Dark Matter Self-interactions and Small Scale Structure. Physics Reports, 730: 1-57.

van den Bosch, F. C., Ogiya, G., Hahn, O. \& Burkert A. (2018). Disruption of dark matter substructure: fact or fiction?. Monthly Notices of the Royal Astronomical Society 474: 3043-3066.

van den Bosch, F. C. \& G. Ogiya. (2018). Dark matter substructure in numerical simulations: a tale of discreteness noise, runaway instabilities, and artificial disruption. Monthly Notices of the Royal Astronomical Society 475: 4066-4087.

Vanderburgh, W. L. (2014). On the interpretive role of theories of gravity and 'ugly' solutions to the total evidence for dark matter. Studies in the History and Philosophy of Modern Physics 47: 62-67.

Vogelsberger, M., Genel, S., Springel, V., Torrey, P., Sijacki, D., Xu, D., ... \& Hernquist, L. (2014). Introducing the illustris project: Simulating the coevolution of dark and visible matter in the universe. Monthly Notices of the Royal Astronomical Society, 444(2): 1518-1547.

Wetzel, A. R., Hopkins, P. F., Kim, J., Faucher-Giguère, C.-A., Kereš, D. \& Quataert, E. (2016). Reconciling Dwarf Galaxies With $\Lambda$ CDM Cosmology: Simulating a Realistic Population of Satellites Around a Milky Way-Mass Galaxy. The Astrophysical Journal, 827(2): L23.

White, S. D. M. \& Rees, M. J. (1978). Core condensation in heavy halos: a two-stage theory for 
galaxy formation and clustering. Monthly Notices of the Royal Astronomical Society, 183(3): 341-358.

Woodward, J. (2003). Making Things Happen: A Theory of Causal Explanation. Oxford University Press.

Worrall, J. (2002). New evidence for old. In P. Gärdenfors, J. Wolenski \& K. Kijania-Placek (Eds.), In the Scope of Logic, Methodology and Philosophy of Science: Volume One of the 11th International Congress of Logic, Methodology and Philosophy of Science, Cracow, August 1999 (pp. 191-209). Springer Netherlands.

Zhu, Q., Marinacci, F., Maji, M., Li, Y., Springel, V. \& Hernquist, L. (2016). Baryonic impact on the dark matter distribution in Milky Way-sized galaxies and their satellites. Monthly Notices of the Royal Astronomical Society, 458(2): 1559-1580.

Zollman, K. J. S. (2010). The Epistemic Benefit of Transient Diversity. Erkenntnis 72: 17-35.

Zwicky, F. (1933/2009). Republication of: The redshift of extragalactic nebulae. General Relativity and Gravitation, 41(1): 207-224. 